\title{
Chemoradiotherapy in octogenarians as primary treatment for muscle-invasive bladder cancer
}

\author{
Victor A. McPherson, MD, MSc; George Rodrigues, MD,2 Glenn Bauman, MD,2 Eric Winquist, MD; ${ }^{3}$ \\ Joseph Chin, MD;1 Jonathan Izawa, MD; Kylea Potvin, MD, ${ }^{3}$ Scott Ernst, MD; Varagur Venkatesan, MD; \\ Tracy Sexton, MD, PhD; ${ }^{2}$ Belal Ahmad, MD; Nicholas Power, $M D^{\prime}$
}

'Division of Urology; '2Division of Radiation Oncology; 3 Division of Medical Oncology; Schulich School of Medicine \& Dentistry, Western University, London, ON, Canada

Cite as: Can Urol Assoc J 2017;11 (1-2):24-30. http://dx.doi.org/10.5489/cuai.4008

See related commentary on page 31.

Visit www.cuai.ca for supplementary data.

\section{Abstract}

Introduction: While radical cystectomy is the gold standard for muscle-invasive bladder cancer (MIBC), in octogenarians cystectomy results in a higher perioperative mortality rate $(6.8-11.1 \%)$ than in younger patients $(2.2 \%)$. Trimodality therapy is a bladdersparing regimen composed of transurethral resection of bladder tumour (TURBT) and chemoradiotherapy, with intent for salvage cystectomy, and has a $62.5-90 \%$ initial complete response rate. In this study, we evaluate TURBT and chemoradiotherapy without salvage cystectomy in medically inoperable octogenarian patients. Methods: We identified a retrospective cohort of patients aged 80-89 years with invasive urothelial carcinoma who received combination chemoradiotherapy between 2008 and June 2014. Outcomes were evaluated by Kaplan-Meier (KM) and Cox regression.

Results: In 40 patients, the mean age was 84.5 years (interquartile range [IQR] 83-86). Seventeen patients received hypofractionated, low-dose radiotherapy (LD) (37.5-40 Gy), while 23 received conventionally fractionated radiotherapy (high-dose [HD]) (50-65 Gy). Mean overall survival (OS) was 20.7 months (IQR 12.75-23.25), while mean recurrence-free survival (RFS) was 13.75 months (IQR 3.75-16.5). Patients receiving HD radiotherapy showed improved OS and local RFS (LRFS) without significant differences in Grade 3-4 toxicities. Univariate Cox regression identified hydronephrosis as a predictor of worse OS and local recurrence and HD radiotherapy as a predictor of improved OS and local recurrence rates. Multivariate Cox regression identified hydronephrosis to be a significant predictor of LRFS.

Conclusions: Primary chemoradiotherapy for inoperable patients with MIBC resulted in a three-year OS of 54.9\% (comparable to cystectomy) and three-year RFS of $42.3 \%$. Superior outcomes were associated with more aggressive chemoradiotherapy treatment. The results of the local control subanalyses in this study are hypothesisgenerating due to the limited patient numbers in the cohort.

\section{Introduction}

Approximately 70000 people are diagnosed with bladder cancer in the U.S. each year ${ }^{1}$ and $90 \%$ of these are urothelial carcinomas (UC). ${ }^{2}$ The gold standard therapy for muscleinvasive UC is radical cystectomy; however, cystectomy bears a Grade 3-5 complication rate of $13 \%{ }^{3}$ and a perioperative mortality of 2.5-5.2\%. ${ }^{3-6}$ Additionally, perioperative mortality increases with age, increasing from $4.7 \%$ in patients in their 60s, to $6.8-11.1 \%$ in octogenarians. 5,7 Even with the additional risks of surgery, population-based evidence suggests local therapy delivered with curative intent improves overall survival (OS) and cancer-specific survival (CSS) in octogenarians. ${ }^{8}$

Trimodality therapy (TMT) is an alternative to cystectomy comprised of transurethral resection of bladder tumour (TURBT), followed by combination chemoradiotherapy, with the intention to have the patient undergo salvage cystectomy if there is local treatment failure. The benefit of combination chemotherapy added to TMT remain unproven, so it typically is not included in this treatment approach. ${ }^{9}$ TMT initial complete response (CR) rates vary from approximately $60-90 \%,{ }^{10-17}$ with five-year bladder intact survival in these studies at 40-45\%, ${ }^{18}$ and an approximate acute Grade 3/4 toxicity rate of $36-46 \%{ }^{14,17}$ and $15.7 \%$ during long-term followup. ${ }^{17}$

At the London Regional Cancer Program, combination chemoradiotherapy has been offered to medically inoperable patients for nearly 20 years. This retrospective study sought to evaluate the outcomes of medically inoperable octogenarians treated with TMT alone at London Health Sciences Centre (LHSC).

\section{Methods}

A comprehensive review of electronic patient charts was completed for patients aged 80-89 year who were deemed 
medically unsuitable to undergo cystectomy for muscleinvasive bladder cancer (MIBC) and thus underwent combination chemoradiotherapy as primary therapy for their disease. In our centre, with few exceptions, medically inoperable patients are treated primarily with chemoradiotherapy regimens. The selection of the chemotherapy is typically based upon the patient comorbidities to allow for drug tolerance. Low-dose (LD) and high-dose (HD) radiotherapy regimens are applied with life-prolonging intent, with both protocols having been seen to be able to produce durable treatment effects in our centre; we have previously published on durable treatment responses using the LD regimen. ${ }^{18}$ The selection of radiotherapy regimen is performed on a caseby-case basis at the discretion of the treating physician and often takes patient general health status into consideration. Radiotherapy is applied to the bladder alone, however, in cases where there are suspicious lymph nodes on imaging, these nodes may be included in the radiation target zone at the discretion of the treating physician and on a caseby-case basis.

Patients evaluated were those who underwent therapy between 2008 and June 2014. These dates were chosen to ensure complete electronic records at the beginning of the period and to allow approximately 12 months of followup data. Treatment-related toxicities were retrospectively assigned according to Common Terminology Criteria for Adverse Events (CTCAE) v4.03. Patients with a small-cell component were excluded, as were patients who underwent salvage cystectomy. Patient survival was censored at recorded date of death or last followup. Recurrence was defined as the date of first imaging or clinical evidence of either local or systemic recurrence. Survival figures are recorded from time of diagnosis, while recurrence figures are recorded from the date of first treatment administration. Statistical analysis was completed using the Medcalc software package (MedCalc Software bvba, Belgium).

\section{Results}

\section{Patient demographics}

Forty patients met the inclusion criteria and their baseline characteristics are summarized in Table 1 . The mean age of patients was 84.5 year (interquartile range [IQR] 83-86), with 28 males and 12 females. Thirty-five patients had T2 disease, four had T3, and one had T4 disease, while three were node-positive. Thirty-one patients were deemed not to be cystectomy candidates due to comorbidity status, while others underwent combination therapy due to advanced age, patient preference, locally advanced disease, and performance status. All patients underwent a TURBT as both a therapeutic and diagnostic procedure, however, only 16 of

\begin{tabular}{|c|c|}
\hline Characteristic or treatment & Number of patients \\
\hline Patient total & 40 \\
\hline Mean age & 84.5 (IQR 83-86) \\
\hline \multicolumn{2}{|l|}{ Sex } \\
\hline $\mathrm{M}$ & 28 \\
\hline $\mathrm{F}$ & 12 \\
\hline \multicolumn{2}{|l|}{ Clinical stage } \\
\hline 2 & $35(87.5 \%)$ \\
\hline 3 & $4(10 \%)$ \\
\hline 4 & $1(2.5 \%)$ \\
\hline $\mathrm{N}+$ & $3(7.5 \%)$ \\
\hline Standard urothelial cell carcinoma & $34(85 \%)$ \\
\hline Urothelial cell carcinoma variant & $6(15 \%)$ \\
\hline Micropapillary & 2 \\
\hline Signet ring & 1 \\
\hline Squamous differentiation & 1 \\
\hline Mixed & 2 \\
\hline \multicolumn{2}{|l|}{ ECOG } \\
\hline 0 & $5(12.5 \%)$ \\
\hline 1 & $25(62.5 \%)$ \\
\hline 2 & $5(12.5 \%)$ \\
\hline 3 & $5(12.5 \%)$ \\
\hline \multicolumn{2}{|l|}{ Reason for no cystectomy } \\
\hline Comorbidities & 31 \\
\hline Advanced age & 3 \\
\hline Locally advanced & 1 \\
\hline Patient preference & 4 \\
\hline Performance status & 1 \\
\hline \multicolumn{2}{|l|}{ Radiation dose } \\
\hline $37.5-40$ Gy & $17(42.5 \%)$ \\
\hline $50-65$ Gy & $23(57.5 \%)$ \\
\hline \multicolumn{2}{|l|}{ Chemotherapy received } \\
\hline Carboplatin & $30(75 \%)$ \\
\hline $5-F U+M M C$ & $7(17.5 \%)$ \\
\hline Cisplatin & $3(7.5)$ \\
\hline \multicolumn{2}{|l|}{ Complete TUR } \\
\hline Complete & $16 / 37(43.2 \%)$ \\
\hline Incomplete & $21 / 37(56.8 \%)$ \\
\hline Not recorded & $3 / 40$ \\
\hline \multicolumn{2}{|l|}{ Hydronephrosis } \\
\hline Present & $12 / 37(32.4 \%)$ \\
\hline Not present & $25 / 37(67.6 \%)$ \\
\hline Not recorded & $3 / 40$ \\
\hline \multicolumn{2}{|l|}{ Baseline comorbidities } \\
\hline Cerebrovascular & 9 \\
\hline Cardiovascular & 19 \\
\hline Respiratory & 10 \\
\hline Chronic kidney disease & 4 \\
\hline Diabetes & 8 \\
\hline Peripheral vascular disease & 2 \\
\hline
\end{tabular}

37 patients that had clear documentation as to the extent of resection underwent a complete resection.

Patients received one of several treatment regimens, encompassing either hypofractionated, LD radiotherapy $(\mathrm{n}=17 ; 37.5-40 \mathrm{~Gy}, 15$ fractions, biologically effective dose $[\mathrm{BED}] 10=47-51, \mathrm{BED} 3=69-76)$ or conventionally fraction- 
ated radiotherapy (HD) ( $\mathrm{n}=23$; 50-65 Gy, 20-33 fractions, BED10 $=63-77, B E D 3=92-105)$. One of three sensitizing chemotherapy regimens were used; carboplatin alone, cisplatin alone, or 5-fluorouracil (5-FU) and mitomycin C (MMC). Given the fact that the hypofractionated regimens were associated with a lower BED, we refer to these regimens as low-dose (LD), and the conventionally fractionated regimens as high-dose (HD). A commonly employed strategy was LD radiotherapy with carboplatin, as our early experience suggested this treatment was well-tolerated and effective in this patient population ${ }^{19}$ and 16 patients received this combination.

\section{Treatment-related toxicities and delayed hematuria}

Retrospectively assessed treatment-related acute toxicities are summarized in Table 2. Nine Grade 3-5 toxicities in patients were recorded - four of these were related to pain and one to cellulitis, none of which were life-threatening. Life-threatening toxicities included one patient who died of sepsis while on therapy and one patient who had a myocardial infarction. There were only three Grade 3-4 genitourinary $(\mathrm{GU})$ toxicities and no gastrointestinal (GI) toxicities. The most common late toxicity documented was hematuria $(n=13)$, with the majority being associated with local recurrence $(n=11)$, and nine of these being Grade 3-4 (Supplementary Table 1; available at www.cuaj.ca). Of the two patients with late bleeding without recurrence (and thus likely attributable to the radiotherapy), the toxicity were Grades 1 and 3, respectively.

\section{Overall patient outcomes}

Objective complete response to therapy, as defined by no visible tumour seen on cystoscopy, was seen in 24 of the 31 $(77.4 \%)$ patients who underwent a surveillance cystoscopy within four months of therapy completion. Imaging studies were completed at the discretion of the clinical team. Patient outcomes are summarized in Figs.1, 2, 3. The three-year OS was $54.9 \%$ (Fig. 1) and 11/14 patients died of their disease. The three-year recurrence-free survival (RFS) was $42.3 \%$ (Supplementary Fig. 1; available at www.cuaj.ca), while the three-year local RFS (LRFS) was $48.7 \%$ (Fig. 2). Disease relapse was typically managed with palliative chemotherapy or supportive measures; salvage cystectomy was not performed.

\section{Subgroup analysis}

Chemotherapy regimens were divided into carboplatin and "non-carbo (NC)" chemotherapies, which included cisplatin and 5-FU and MMC. There was no difference in complete response rates between chemotherapy regimens (Supplementary Table 2; available at www.cuaj.ca; Fisher exact test [FET]). Using Kaplan-Meier survival analysis, NC chemotherapy regimens were found to be associated with improved OS by log-rank test (Fig. 3; $p=0.0425$ ). NC chemotherapies also showed a non-significant trend towards improvement in LRFS (Supplementary Fig. 2; available at $w w w . c u a j . c a ; p=0.1119)$. Finally, there was a non-significant trend towards higher rates of Grade 1-2 and 3-5 toxicities in patients who received carboplatin (Supplementary Table 3; available at www.cuaj.ca).

Radiation therapy was divided into LD radiotherapy (37.5-40 Gy) and HD radiotherapy (50-65 Gy). There was no difference in complete response rates between radiotherapy regimens (Supplementary Table 2). HD radiotherapy was found to show an improvement in both OS $(p=0.0348)$ and LRFS ( $p=0.0367)$ by log-rank test (Figs. 4, 5). The HD radiotherapy group did show a higher rate (20/23 vs. 7/17) of Grade 1-2 toxicities vs. the LD radiotherapy group (Table 3 ), however, there were no significant differences in the rates of local regional GI or GU Grade 3-4 toxicities or in the overall rate of delayed hematuria (Table 3 ).

Finally, outcomes were analyzed by chemoradiotherapy regimen. Although limited by the small numbers in each group, the HD, NC group showed a non-significant trend towards improved OS (Fig. 6) and LRFS (Supplementary Fig. 3; available at www.cuaj.ca) when compared with the HD and carboplatin, LD and carboplatin, and LD NC groups.

\section{Cox regression analysis}

Univariate Cox regression analysis was completed to evaluate the contribution of clinical factors to OS and LRFS.

\begin{tabular}{|c|c|c|c|c|c|c|c|c|}
\hline Grade & GI & GU & Cardiovascular & Infectious & Respiratory & Renal & Other & Total \\
\hline 1 & 15 & 15 & 2 & 0 & 1 & 1 & 4 & 38 \\
\hline 2 & 1 & 4 & 0 & 0 & 0 & 0 & 5 & 11 \\
\hline 3 & 0 & 3 & 1 & 1 & 0 & 0 & 2 & 6 \\
\hline 4 & 0 & 0 & 1 & 0 & 0 & 0 & 0 & 1 \\
\hline 5 & 0 & 0 & 0 & 1 & 0 & 0 & 0 & 1 \\
\hline Total & 16 & 22 & 4 & 2 & 1 & 1 & 11 & 58 \\
\hline Total Grade 3-5 & 0 & 3 & 2 & 2 & 0 & 0 & 2 & 9 \\
\hline
\end{tabular}




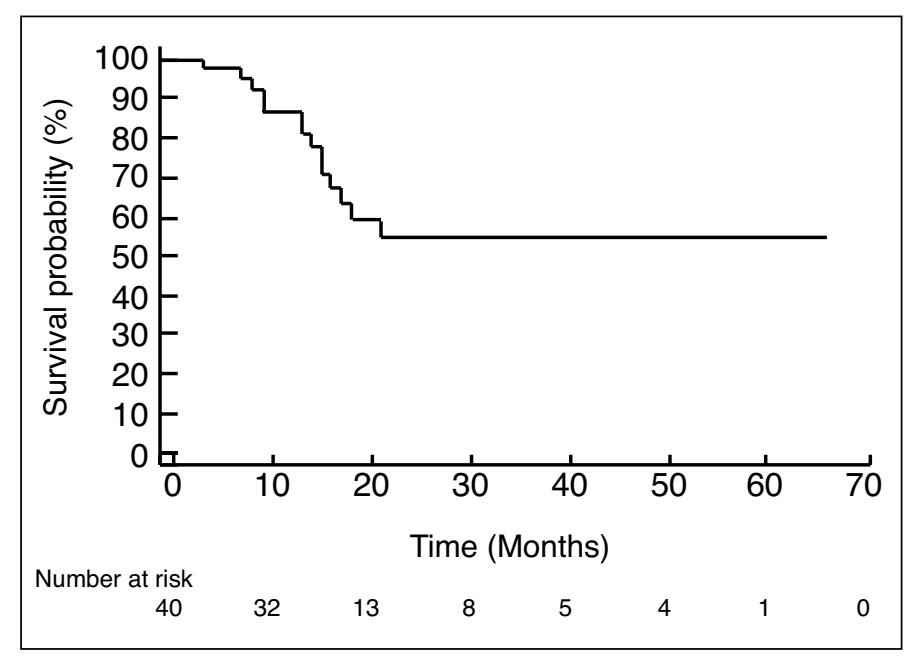

Fig. 1. Overall cohort: Kaplan-Meier overall survival.

Hydronephrosis (hazard ratio [HR] 3.13, 95\% confidence interval $[\mathrm{Cl}]$ 1.10-8.92) and HD radiotherapy (HR 0.34, 95\% $\mathrm{Cl}$ 0.12-0.97) were significant predictors of OS (Table 4), while hydronephrosis (HR 4.67, 95\% Cl 1.53-14.31) and HD radiotherapy (HR $0.35,95 \% \mathrm{Cl} 0.13-0.99$ ) were significant predictors of LRFS (Table 5).

Multivariate Cox regression was also completed using the variables that were found to be statistically significant on univariate analysis. This found only a trend towards significance for both hydronephrosis and HD radiotherapy in OS (Table 4), while hydronephrosis (HR 3.95, 95\% Cl 1.29-12.1) was a predictor of LRFS (Table 5).

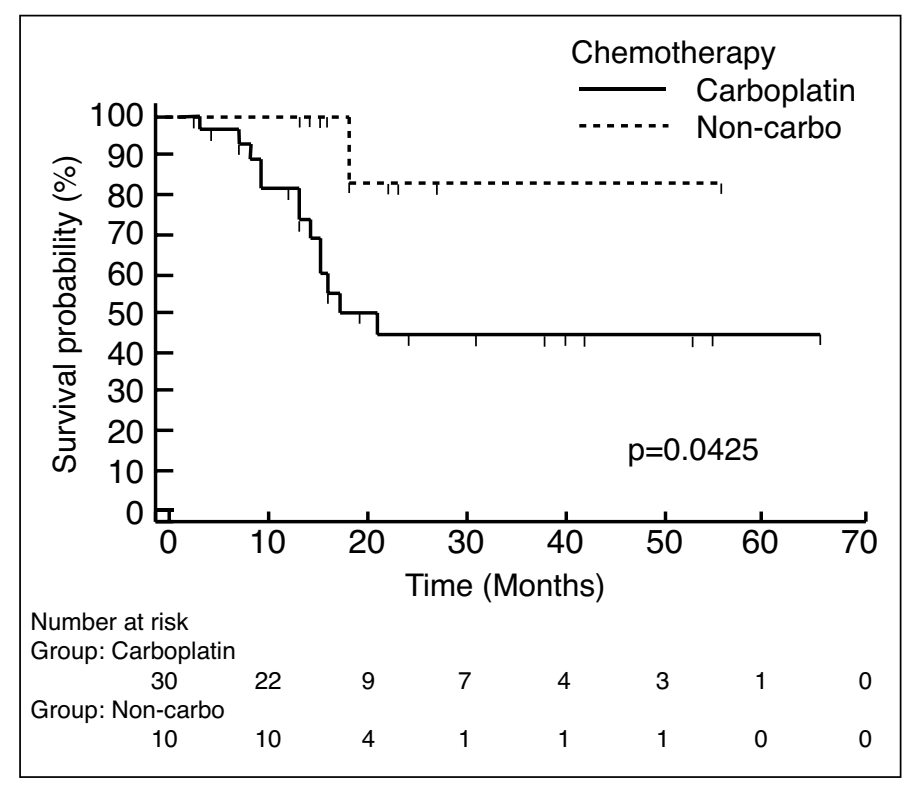

Fig. 3. Kaplan-Meier overall survival analysis by chemotherapy regimen.

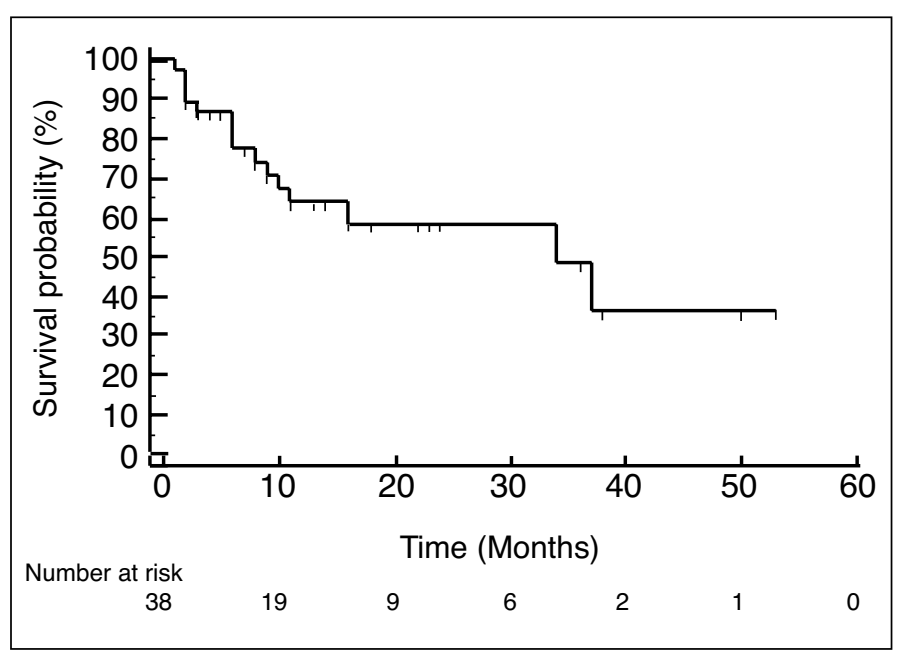

Fig. 2. Overall cohort: Kaplan-Meier local recurrence-free survival.

\section{Discussion}

In this retrospective review of 40 patients aged 80-89 years (mean 84.5) who were medically inoperable for MIBC and treated with chemoradiotherapy, the three-year OS, RFS, and LRFS were $54.9 \%, 42.3 \%$, and $48.7 \%$, respectively. The historical results of radical cystectomy, indicate fiveyear OS and LRFS figures of $40-60 \%$ and $80-90 \%$, respectively. ${ }^{18}$ Octogenarians have a median survival of only 3.3 years following radical cystectomy, compared with 7.7 years for their younger counterparts, and have a $42.3 \%$ five-year cumulative death rate from other causes. ${ }^{5}$ Thus, our threeyear OS outcomes are comparable for reported survival for octogenarians undergoing cystectomy and the requirements

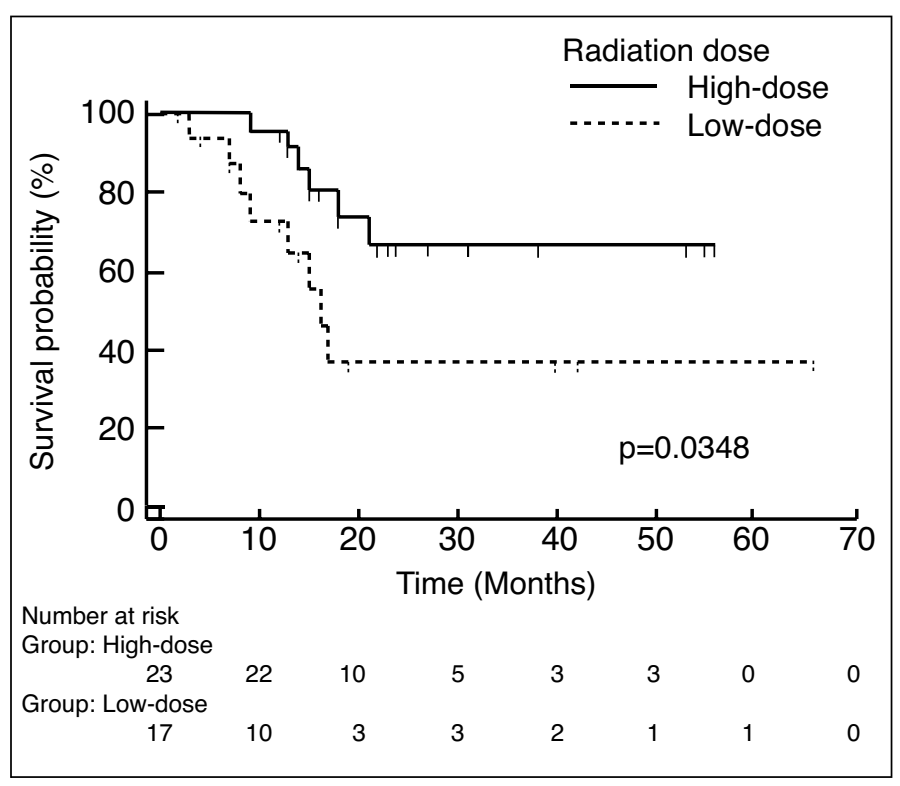

Fig. 4. Kaplan-Meier overall survival analysis by radiotherapy regimen. 


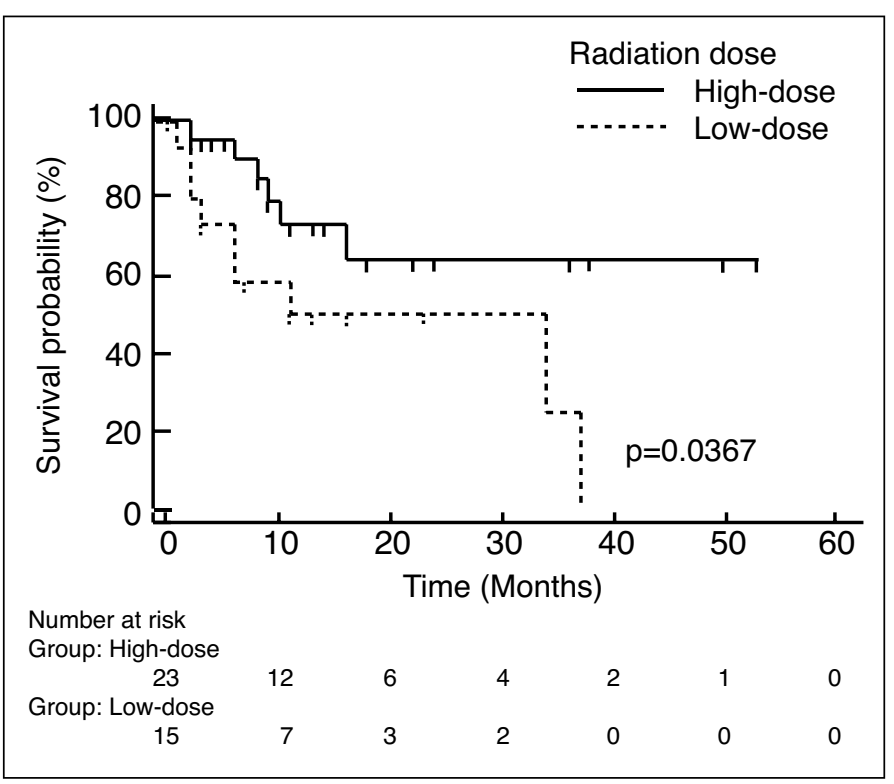

Fig. 5. Kaplan-Meier local recurrence-free survival analysis by radiotherapy regimen.

for long-term disease control in this patient population is less stringent due to the lower median survival time.

Radical cystectomy bears significant morbidity and mortality, with an overall perioperative complication rate of $28-49 \%,{ }^{3,4}$ and a Grade 3-5 complication rate of approximately $13 \% .^{3-6}$ Additionally, perioperative mortality rates rise in octogenarians, ${ }^{5}$ and while overall Grade 3-5 complications are similar, one study identified a significant increase in cardiovascular (9.5-19.7\%) and neurological (3.9-10.3\%) complications. ${ }^{5}$ In our study, there were nine Grade 3-5 acute toxicities $(22.5 \%)$, with one patient who died from sepsis while on treatment $(2.5 \%)$ and one who suffered a non-fatal myocardial infarction. Acute Grade 1-2 GU (19; $47.5 \%)$ and GI $(16 ; 40 \%)$ toxicities were common, while severe late toxicities were uncommon, with only two patients experiencing late treatment-related hematuria, of which one

\begin{tabular}{lcc}
\hline Table 3. Local toxicities by radiation dose & \\
\hline Toxicity & $\begin{array}{c}\text { Proportion of } \\
\text { patients affected }\end{array}$ & p (FET) \\
\hline Grade 1-2 toxicities & $7 / 17(41.2 \%)$ & \\
37.5-40 Gy & $20 / 23(87.0 \%)$ & $\mathbf{0 . 0 0 5 1 *}$ \\
50-65 Gy & & \\
Grade 3-4 toxicities & $1 / 17(5.9 \%)$ & 0.4250 \\
37.5-40 Gy & $0 / 23(0 \%)$ & \\
50-65 Gy & & \\
Delayed hematuria & $5 / 17(29.4 \%)$ & 0.2499 \\
$37.5-40$ Gy & $3 / 23(13.0 \%)$ & \\
50-65 Gy & & \\
Overall; Grade 3-4+ hematuria & $5 / 17(29.4 \%)$ & \\
37.5-40 Gy & $3 / 23(13.0 \%)$ & 0.2499 \\
\hline $50-65$ Gy & & \\
\hline FET: Fisher exact test. & & \\
\hline
\end{tabular}

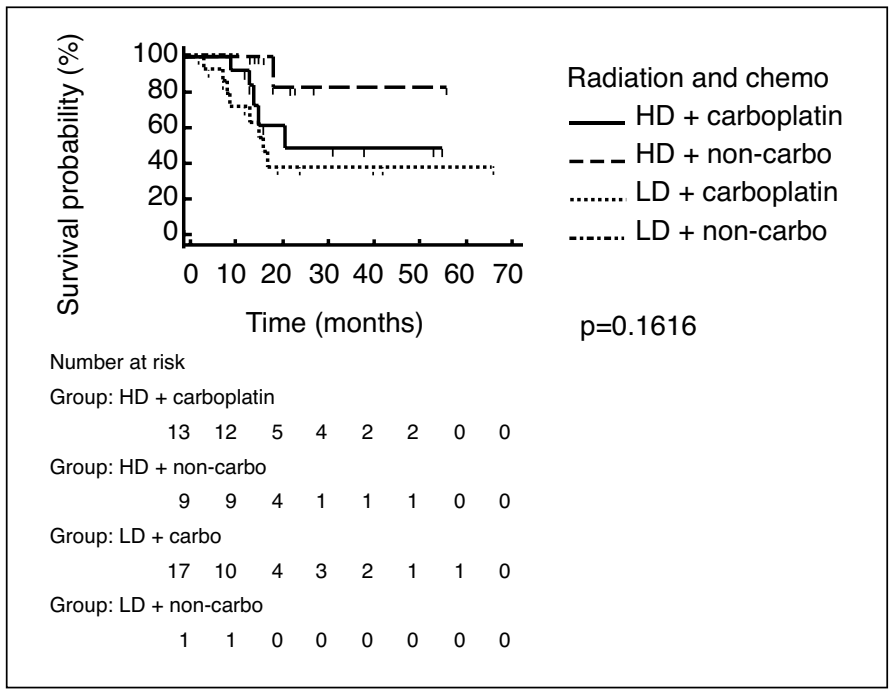

Fig. 6. Kaplan-Meier overall survival analysis by chemoradiotherapy regimen.

patient had Grade 1 toxicity and the other Grade 3 (11 had late bleeding related to tumour recurrence). Therefore, the octogenarian mortality rate in our study was lower than that in the cystectomy literature $(2.5 \%$ vs. $6.8-11.1 \%),{ }^{5}$ while there was an increased rate of Grade 3-5 complications; most, however, were non-life-threatening.

In this study, the choice of chemotherapy regimen was associated with improved OS, but not LRFS. Carboplatin may have been given to patients with multiple comorbidities, reduced performance status, and/or impaired renal function precluding the use of cisplatin; therefore, this may represent a selection bias. For example, patients with hydronephrosis, an adverse prognostic factor, may be more likely to receive carboplatin because of impaired renal function. That said, a phase 3 study of 360 patients given 5-FU and MMC showed a $13 \%$ improvement in both two-year locoregional disease-free survival (DFS) and in five-year OS

Table 4. Cox regression analysis predicting survival

\begin{tabular}{lccc}
\hline Unvariable analysis & Hazard \\
Variable & $\begin{array}{c}\text { 95\% confidence } \\
\text { interval }\end{array}$ & $\mathbf{p}$ \\
\hline Hydronephrosis & 3.13 & $1.10-8.92$ & $\mathbf{0 . 0 3 3 9 *}$ \\
Complete TUR & 0.89 & $0.31-2.55$ & 0.8256 \\
Cardiovascular disease & 2.63 & $0.91-7.63$ & 0.0749 \\
Respiratory disease & 1.54 & $0.52-4.58$ & 0.4493 \\
Diabetes & 0.30 & $0.04-2.25$ & 0.1644 \\
Nodal disease & 1.30 & $0.17-9.89$ & 0.8106 \\
High-dose radiation & 0.34 & $0.12-0.97$ & $\mathbf{0 . 0 4 5 7 *}$ \\
Non-carbo & 0.16 & $0.02-1.22$ & 0.0786 \\
\hline Multivariable analysis & \multicolumn{3}{l}{} \\
\hline Hydronephrosis & 2.82 & $0.98-8.15$ & 0.0569 \\
High-dose radiation & 0.41 & $0.14-1.21$ & 0.1072 \\
\hline *Statistically significant. TUR: transurethral resection. &
\end{tabular}




\begin{tabular}{|c|c|c|c|}
\hline \multicolumn{4}{|l|}{ Univariable analysis } \\
\hline Variable & Hazard ratio & $\begin{array}{c}95 \% \\
\text { confidence } \\
\text { interval }\end{array}$ & $\mathbf{p}$ \\
\hline Hydronephrosis & 4.67 & $1.53-14.31$ & $0.0083 *$ \\
\hline Complete TUR & 0.81 & $0.29-2.29$ & 0.6960 \\
\hline High-dose radiation & 0.35 & $0.13-0.99$ & $0.0458 *$ \\
\hline Non-carbo & 0.32 & $0.0723-1.42$ & 0.0906 \\
\hline \multicolumn{4}{|l|}{ Multivariable analysis } \\
\hline Hydronephrosis & 3.95 & $1.29-12.1$ & $0.0168 *$ \\
\hline High-dose radiation & 0.46 & $0.16-1.33$ & 0.1562 \\
\hline
\end{tabular}

vs. radiotherapy alone ${ }^{17}$ and may be a more appropriate alternative to carboplatin when cisplatin regimens are not tolerated. However, the approximate four weeks required to fully deliver this chemotherapy regimen would limit its applicability in patients selected for shorter-term, LD radiotherapy regimens.

LD radiotherapy with platinum chemosensitization has been administered in our centre in order to provide disease control while minimizing toxicity and overall treatment time in frail patients, as previously reported. ${ }^{19}$ In this current study, while a reduced low-grade toxicity was noted in the LD radiotherapy group, outcomes in terms of OS and LRFS were inferior, while there was no difference in the number of Grade 3-4 toxicities or hematuria. This corresponds with the result of a 2006 Dutch study, which showed that 10 Gy increments in the final radiotherapy dose increased the local control rate by $50 \%$ in three years. ${ }^{20,21}$ Notably, while there is evidence regarding the overall radiation dose, studies have shown no effect of the number of fractions or duration of treatment on local control, and thus hypofractionated (55 Gy/20 fractions) gives comparable results to conventionally fractionated (62 Gy/32 fractions) radiotherapy. ${ }^{21,22}$

This study has a number of potential limitations. It is a retrospective analysis with a limited number of heterogeneously treated patients, limiting its statistical power. All outcomes, including adverse events, recurrence, and death were assessed retrospectively, and causality of adverse events was inferred from the available clinical documentation, with variable number of followup visits surrounding therapy. Recurrence and death were determined from imaging tests and clinical notes, and not all patients underwent a post-treatment cystoscopy for assessment of tumour response. Finally, no adjustment was made in the Cox regression analysis to account for multiple comparisons due to the limited sample size of our study. Thus, we acknowledge that the results are primarily hypothesis-generating.

However, the results are consistent with conclusions that chemoradiotherapy is a safe treatment option for MIBC in octogenarians and is associated with three-year OS and RFS rates of $54.9 \%$ and $42.3 \%$, respectively in our cohort. Based on our experience and reports of prospective trials, our results with TMT comprised of TURBT followed by hypofractionated HD radiotherapy (55 Gy/20 fractions) with concurrent 5-FU and MMC, as described in the study by James et al in $2012^{17}$ appear to provide an appropriate balance between risk of toxicity and optimizing clinical outcomes in suitable octogenarians.

Competing interests: Dr. Bauman has received clinical trial funding from Sanofi. Dr. Winquist has participated in clinical trials for Roche and Sanofi. Dr. Chin has been an advisor for US HIFU and Profound Medical Inc. Dr. Izawa has received speaker honoraria from Astellas, AstraZeneca, Janssen, and Sanofi. Dr. Ernst has been an advisor for Astellas, BMS, Merck, Novartis, and Roche; a speaker for BMS, Novartis, and Roche; and has participated in clinical trials for Astellas, BMS, Janssen, Merck, Roche, and Tokai. Dr. Ahmad has received honoraria from Ferring and has participated in several radiation studies for prostate, bladder, and kidney cancers for the London Regional Cancer Program. Dr. Power has participated in clinical trials for Argos Therapeutics. The remaining authors report no competing personal or financial interests.

This paper has been peer-reviewed.

\section{References}

1. Jemal A, Siegel R, Xu J, et al. Cancer statistics, 2010. CA Cancer J Clin 2010;60:277-300. https://doi.org/10.3322/caac.20073

2. Parkin DM, Bray F, Ferlay J, et al. Global cancer statistics, 2002. CA Cancer J Clin 2005;55:74-108. https://doi.org/10.3322/caniclin.55.2.74

3. Novara G, De Marco V, Aragona $M$, et al. Complications and mortality after radical cystectomy for bladder transitional cell cancer. J Urol 2009;182:914-21. https://doi.org/10.1016/i.juro.2009.05.032

4. Stein JP, Lieskovsky $G$, Cote R, et al. Radical cystectomy in the treatment of invasive bladder cancer: Long-term results in 1054 patients. J Clin Oncol 2001;19:666-75.

5. Hautmann RE, Gschwend JE, de Petriconi RC, et al. Cystectomy for transitional cell carcinoma of the bladder: Results of a surgery-only series in the neobladder era. J Urol 2006;176:486-92. https://doi.org/10.1016/i.juro.2006.03.038

6. Hautmann RE, de Petriconi RC, Pfeiffer C, et al. Radical cystectomy for urothelial carcinoma of the bladder without neoadjuvant or adjuvant therapy: Long-term results in 1100 patients. Eur Urol 2012;61:1039-47. https://doi.org/10.1016/i.eururo.2012.02.028

7. Donat SM, Siegrist T, Cronin A, et al. Radical cystectomy in octogenarians — does morbidity outweigh the potential survival benefits? J Urol 2010;183:2171-7. https://doi.org/10.1016/i.juro.2010.02.015

8. Hollenbeck BK, Miller DC, Taub D, et al. Aggressive treatment for bladder cancer is associated with improved overall survival among patients 80 years old or older. Urology 2004;64:292-7. https://doi.org/10.1016/i.urology.2004.03.034

9. Shipley WU, Winter KA, Kaufman DS, et al. Phase 3 trial of neoadjuvant chemotherapy in patients with invasive bladder cancer treated with selective bladder preservation by combined radiation therapy and chemotherapy: Initial results of Radiation Therapy Oncology Group 89-03. J Clin Oncol 1998; 16:3576-83.

10. Sangar VK, McBain CA, Lyons J, et al. Phase 1 study of conformal radiotherapy with concurrent gemcitabine in locally advanced bladder cancer. Int I Radiat Oncol Biol Phys 2005;61:420-5. hitps://doi.org/10.1016/i.i.irobp.2004.05.074

11. Oh KS, Soto DE, Smith DC, et al. Combined-modality therapy with gemcitabine and radiation therapy as a bladder preservation strategy: Long-term results of a phase 1 trial. Int J Radiat Oncol Biol Phys 2009;74: 511-7. https://doi.org/10.1016/i.ijrobp.2008.08.021

12. Choudhury A, Swindell R, Logue JP, et al. Phase 2 study of conformal hypofractionated radiotherapy with concurrent gemcitabine in muscle-invasive bladder cancer. J Clin Oncol 2011;29:733-8. https://doi.org/10.1200/JC0.2010.31.5721 
McPherson et al.

13. Atasoy BM, Dane F, Alsan Cetin I, et al. Concurrent chemoradiotherapy with low-dose weekly gemcitabine in medically inoperable muscle-invasive bladder cancer patients. Clin Transl Oncol 2014;16:91-5. https://doi.org/10.1007/sl2094-013-1047-8

14. Rödel C, Grabenbauer GG, Kühn R, et al. Combined-modality treatment and selective organ preservation in invasive bladder cancer: Long-term results. J Clin Oncol 2002;20:3061-71. https://doi.org/10.1200/ JC0.2002.11.027

15. Danesi DT, Arcangeli G, Cruciani E, et al. Conservative treatment of invasive bladder carcinoma by transurethral resection, protracted intravenous infusion chemotherapy, and hyperfractionated radiotherapy. Cancer 2004;101:2540-8. https://doi.org/10.1002/cncr.20654

16. Efstathiou JA, Spiegel DY, Shipley WU, et al. Long-term outcomes of selective bladder preservation by combined-modality therapy for invasive bladder cancer: The MGH experience. Eur Urol 2012;61:705-11 https://doi.org/10.1016/i.eururo.2011.11.010

17. James ND, Hussain SA, Hall E, et al. Radiotherapy with or without chemotherapy in muscle-invasive bladder cancer. N Engl J Med 2012;366:1477-88. https://doi.org/10.1056/NEJMoal 106106
18. Mak RH, Zietman AL, Heney NM, et al. Bladder preservation: Optimizing radiotherapy and integrated treatment strategies. BJU Int 2008; 102:1345-53. https://doi.org/10.1111/j.1464-410X.2008.07981.x

19. Greenland JD, Bauman GS, Winquist E, et al. Palliative chemoirradiation for transitional carcinoma of the bladder: A case report. Can J Urol 2000;7:1085-7.

20. Zhang $S$, Yu YH, Zhang $Y$, et al. Radiotherapy in muscle-invasive bladder cancer: The latest research progress and clinical application. Am I Cancer Res 2015;5:854-68.

21. Pos FJ, Hart G. Schneider C, et al. Radical radiotherapy for invasive bladder cancer: What dose and fractionation schedule to choose? Int J Radiat Oncol Biol Phys 2006;64:1168-73. https://doi.org/10.1016/i. ïrobp.2005.09.023

22. Moonen L, vd Voet H, de Niis R, et al. Muscle-invasive bladder cancer treated with external beam radiation: Influence of total dose, overall treatment time, and treatment interruption on local control. Int I Radiat Oncol Biol Phys 1998;42:525-30. https://doi.org/10.1016/S0360-3016(98)00263-6

Correspondence: Dr. Nicholas Power, Division of Urology, Schulich School of Medicine \& Dentistry, Western University, London, ON, Canada; Nicholas.Power@hhsc.on.ca 